REVISTA INTERNACIONAL DE CIENCIAS DEL DEPORTE International Journal of Sport Science

doi:10.5232/ricyde2005.00103

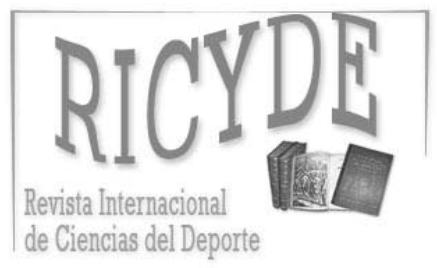

International Journal of Sport Science VOLUMEN I. AÑO I

Páginas: 28-45 ISSN: 1885-3137 No 1 - Octubre - 2005

\title{
Comportamiento Motor Espontáneo en el Patio de Recreo Escolar: Análisis de las diferencias por género en la ocupación del espacio durante el recreo escolar. \\ Spontaneous motor behaviour in school recess: Analysis of gender differences in the space use.
}

\author{
Ramón Cantó Alcaraz \\ Facultad de Ciencias de la Actividad Física y del Deporte. INEF \\ Universidad Politécnica de Madrid \\ Luis Miguel Ruiz Pérez \\ Facultad de Ciencias del Deporte \\ Universidad de Castilla la Mancha. Toledo
}

Resumen

\begin{abstract}
El propósito de este estudio es analizar la existencia de comportamientos motrices diferenciados entre chicos y chicas, así como las posibles causas ambientales-físicas que provocan, o facilitan, el mal reparto del espacio disponible en el patio de recreo escolar. De manera específica este estudio tiene por objeto valorar el reparto del espacio disponible en el patio de recreo escolar, entre un grupo de escolares a lo largo de un curso escolar. Por medio de una metodología observacional se han identificado y constatado los distintos espacios empleados en cada una de las Categorías
\end{abstract}

comportamentales establecidas, con la finalidad de poder establecer la territorialidad (reparto, ocupación y uso) entre los niños y las niñas participantes en este estudio, dentro del espacio disponible en el patio de recreo escolar. El análisis de las observaciones registradas, mostraron la existencia de dichas desigualdades en el uso y empleo del espacio disponible, desigualdad que perjudica a las niñas. El estudio concluye con la necesidad de repensar los espacios de juego para que tanto niños como niñas puedan desplegar sus posibilidades de movimiento en el momento del recreo escolar.

Palabra clave: espacio escolar, erquitectura escolar, patios escolares, territorialidad, género, comportamiento motor.

Correspondencia: Dr. Ramón Cantó Alcaraz

Facultad de Ciencias de la Actividad Física y del Deporte-INEF. Universidad Politécnica de Madrid C/ Martín Fierro s/n 28040-Madrid

email: r.canto@upm.es 
REVISTA INTERNACIONAL DE CIENCIAS DEL DEPORTE International Journal of Sport Science

No 1

Fecha: Octubre de 2005

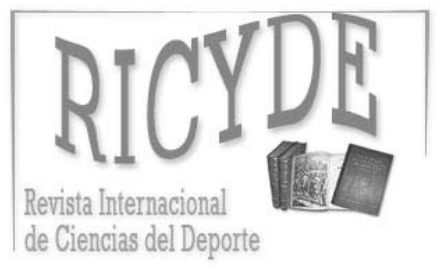

International Journal of Sport Science VOLUMEN I. AÑO I

Páginas: 28-45 ISSN: 1885-3137 No 1 - Octubre - 2005

\title{
Spontaneous motor behaviour in school recess: Analysis of gender differences in the space use.
}

\section{Comportamiento Motor Espontáneo en el Patio de Recreo Escolar: Análisis de las diferencias por género en la ocupación del espacio durante el recreo escolar.}

\author{
Ramón Cantó Alcaraz \\ Facultad de Ciencias de la Actividad Física y del Deporte. INEF \\ Universidad Politécnica de Madrid \\ Luis Miguel Ruiz Pérez \\ Facultad de Ciencias del Deporte \\ Universidad de Castilla la Mancha. Toledo
}

\begin{abstract}
The purpose of this study is to analyse the existence of diverse motor behaviour between boys and girls and the possible physical environmental factors which may cause or facilitate the poor distribution of the available playground space. Through a methodical approach, the aim of the study is to assess the distribution of the allocated round by monitoring a group of students over the course of the school year. Different spaces used in each of the established behavioural categories have been identified and

verified through a strict observation process. The objective being to establish the territorial boundaries of the space being used by the boys and girls. The analysis of the observations showed an existence of inequality in the available space prejudicing the girls. The study concludes with the necessity of a complete re-think towards the respective play area so that the boys and the girls can display equally their possibilities of movement during the break/playtime.
\end{abstract}

Key words: School space, school architecture, school recess, territoriality motor behaviour. 


\section{Introducción}

El punto de partida de este estudio está incluido o podemos ubicarlo en el contexto de los llamados comportamientos diferenciados hombre/mujer y la discusión que todo ello suscita, y que, en algunos contextos, se ha venido en llamar crisis de género ó sexista. En este estudio consideramos la evidente adjudicación de conductas motrices diferenciadas para cada uno de los géneros, contemplándose un cierto número de ellas reforzadas para uno de los dos. Este estudio se circunscribe en un lugar y tiempo, no pasamos por alto que los criterios diferenciadores de estas conductas van cambiando con el paso del tiempo y no son iguales en todas las sociedades o culturas.

En los últimos tiempos, como consecuencia de los cambios sociales y la apertura ideológica, se ha avanzado hacia una concepción no discriminadora del sexo. Como indican García y Asins (1994, p.2): “La segunda mitad del siglo XX es el período en el cual las mujeres han adquirido una presencia cada vez mayor en campos y actividades que hasta entonces, habían sido dominio exclusivo de los varones. Una breve mirada al camino que tuvieron que recorrer y a los obstáculos que hubieron de salvar, nos puede ayudar a comprender por qué en la actualidad, pese a los grandes cambios acontecidos que han afectado profundamente al universo femenino, existen muchos comportamientos, actitudes y valoraciones de marcado carácter sexista."

Sin embargo, una y otra vez la discusión llega tarde, hecho que se repite generación tras generación, cada vez que ellos y ellas se incorporan al contexto en el que se ponen de manifiesto las diferencias (nos referimos a mundo laboral, político, familiar, etc.), olvidando que los niños y las niñas necesitan prepararse para desempeñar roles nuevos en función de las circunstancias cambiantes y estar dispuestos a cambiar de roles en el transcurso de su vida.

Los contactos que se realizan en la primera infancia son moldeadores de conductas variadas (alejadas de los estereotipos lo más posible) y flexibles, adaptadas a estos futuros contextos socioculturales. Según Pomerleau (1992), la diferenciación sexual de los comportamientos se moldea desde la cuna. Y no olvidemos que los actuales objetivos del desarrollo de niños y de niñas difieren de lo que fueron antaño, y exigen cada vez más de modificaciones en todos los aspectos y prácticas educativas y en el diseño de la infraestructura de los entornos educativos (edificios, aulas, zonas de recreo, etc.). Sería suficiente para reforzar esta afirmación con contemplar el diseño de distribución de espacios escolares, donde aulas y zonas de recreo mantienen desde hace muchos años, y que induce, pensamos y trataremos de demostrar, a un desigual reparto del espacio donde, sin duda beneficia a los varones.

Hace años que venimos contemplando como un alto porcentaje de las zonas de recreo escolar, es ocupado por campos de fútbol, quedando ciertas zonas reducidas para la concentración de niñas, evidenciando una diferencia, al menos visual, en cuanto a la densidad de niños y niñas por metro cuadrado. Con ello queremos inicialmente plantear y ponerles en contacto con nuestra preocupación, inductora en este caso de nuestro estudio, y responder entre otras a cuestiones tales como: ¿'son las instituciones escolares con sus diseños para la ocupación y uso de los espacios, responsables en parte del problema que nos ocupa? 
La observación cotidiana nos muestra que a pesar del consenso, o acuerdo explícito, en materia de igualdad de oportunidades en la educación, ciertos espacios o territorialidades urbanístico / educativas permanecen invariables desde hace décadas, invitándose a la reproducción de conductas estereotipadas en niños y niñas, que no facilitan la citada igualdad. Sin ir más lejos, pueden contemplarse patios de recreo escolares, cuyos criterios de diseño aparecen invariables año tras año, al menos así lo vemos desde nuestra permanente actuación en ellos como educadores.

Tratamos de establecer si un entorno como el patio de recreo escolar, diversificado con arreglo a otros criterios (que no perpetúen estereotipos motores de género) facilitaría conclusiones dispares o diferentes, a la hora de analizar el comportamiento de niños y niñas. Una dimensión de la persona que refleja el efecto del proceso de socialización, es el comportamiento motor, y a partir de él la ocupación y uso de espacio disponible (el reparto entre ambos géneros del patio de recreo) de ahí que nuestro interés se centre en dicho comportamiento en un contexto tan institucionalizado como es el del patio de recreo (contemplado como medio natural válido de observación), y en situación espontánea.

\section{Planteamiento del problema}

El problema planteado en este estudio está referido a analizar las diferencias existentes en la ocupación y uso el espacio en un grupo de escolares, niño y niñas, de 8-9 años en un centro que se podría considerar prototípico de los centros escolares que existen en España.

De manera específica este estudio tiene por objeto, describir, clasificar, y analizar el repertorio de comportamientos motrices producto de las relaciones interpersonales en el tiempo de recreo, y en concreto: Identificar y constatar los distintos espacios empleados en cada una de las categorías conductuales, con el fin de poder establecer un medio de cuantificar la territorialidad (reparto, ocupación y uso) en el patio de recreo escolar.

\section{Método}

\section{Participantes}

En este estudio fueron observados en el patio de recreo 29 escolares (15 niñas y 14 niños). Al inicio del curso escolar su edad era de 8 años pero que en el transcurso de la fase activa del estudio observacional, todos cumplieron los 9 años de edad. Todos los permisos necesarios para poder realizar el estudio fueron solicitados tanto a los responsables del centro como a los padres o tutores de los escolares participantes.

La muestra pertenece a una población homogénea de clase media alta que han estado escolarizados juntos desde los 3 años en el mismo centro hasta el momento del estudio. Por lo tanto, esta muestra manifiesta una distribución binomial, es decir, tiene una distribución muestral de las proporciones observadas en una muestra tomada al azar, o disponible, de una población de dos clases. Todas las familias de los componentes del grupo poseen un status económico y socio-cultural similar (medio-alto), teniendo en cuenta el costo académico de la escolaridad de los niños, resaltando que en el centro los estudios se realizan con enseñanza bilingüe (inglés-español), desde los 3 años. 
Cantó, R. y Ruiz, L. M. (2005) Comportamiento Motor Espontáneo en el Patio de Recreo Escolar: Análisis de las diferencias por género en la ocupación del espacio durante el recreo escolar. Revista Internacional de Ciencias del Deporte. 1 (1), 28-45. http://www.cafyd.com/REVISTA/art3n1a05.pdf

\section{Procedimiento}

Para llevar a cabo el presente estudio se empleo una metodología observacional en la que se realizaron registros sobre una plantilla de categorización de conductas que previamente había sido establecida (Cantó 2004).

\section{- $\quad$ Fases de la observación}

El procedimiento utilizado en este estudio constó de las siguientes etapas:

$1^{a}$. Fase de observación-reflexión: Fase de puesta a punto de la estrategia.

$2^{a}$. Fase de observación activa: Observación sistemática: Justificación y problemas del procedimiento

En la primera fase se ha procedido a través de un largo período de observación - reflexión a observar a la muestra durante 5 años, (durante los cursos académicos 1991 a 1996) fase, por tanto, de observación informal, con registros narrativos, que permitieron contar con los criterios necesarios con los que llevar a cabo la puesta a punto de la estrategia de investigación.

En una segunda fase se realizó una observación activa y sistemática al objeto de cubrir los objetivos establecidos en el estudio. Dicha observación se llevó a cabo, dos veces por semana durante un curso escolar (5 meses, de Febrero a Junio de 1996) a la misma hora, esto es, en el segundo recreo de la mañana, a 12.15 horas los mismos días de la semana alternos, y bajo las mismas circunstancias materiales de observación.

El total de sesiones de observación fue de 40 sesiones, a razón de dos por semana durante 5 meses. (La mitad +1 del total de la duración del curso escolar.) Cabe destacar que si bien el tiempo de recreo transcurría de 12,00 a 13,00 horas, el tiempo dedicado al registro fue el comprendido entre las 12, 20 y las 12, 40 horas durante las 40 sesiones. (Cuadro 1)

Cuadro 1. Resumen de pautas de la fase de observación activa

\section{o 40 sesiones de observación de 20 minutos cada una}

o Sesiones distribuidas a razón de dos por semana durante 5 meses, los comprendidos de Febrero a Junio de 1996)

o En horario comprendido entre las 12.20 horas a 13.45 horas

o Un total de 800 minutos

o A estas 40 hojas de registro hay que sumar otras 20 completadas por los observadorescolaboradores, que durante 10 sesiones elegidas al azar, simultanearon un total de dos observadores por sesión, al efecto de utilizar el $25 \%$ de las sesiones ${ }^{1}$ de observación para aplicar la fórmula de fiabilidad, que evaluara al máximo el posible sesgo existente. 
Cantó, R. y Ruiz, L. M. (2005) Comportamiento Motor Espontáneo en el Patio de Recreo Escolar: Análisis de las diferencias por género en la ocupación del espacio durante el recreo escolar. Revista Internacional de Ciencias del Deporte. 1 (1), 28-45. http://www.cafyd.com/REVISTA/art3n1a05.pdf

\section{Instrumentos}

Se aplicó una plantilla / hoja de registros de conductas categorizadas previamente diseñada y validada. En cuanto al diseño de la hoja de registros, lo primero que exige una investigación observacional, es la elaboración de un esquema de codificación, esto es algo que nos ha llevado mucho tiempo, años de observación informal o no sistemática, registros narrativos, y finalmente tras innumerables intentos de reagrupamiento de tipos de conductas, un esquema codificado de conductas objetivo, que definidas de antemano varias modalidades de conducta - sistema de categorías - y solicitar entonces a los observadores que registren, cada vez que ocurra la conducta, el correspondiente código predefinido.

El sistema de categorías que exponemos responde a la laboriosa y ardua tarea de agrupamiento de tipos de conductas desarrollada con el grupo de niños observados, filmados y fotografiados durante la fase pasiva, de forma prolongada y asidua por el investigador principal, y de forma colegiada, con los miembros del equipo de observadores durante los períodos de entrenamiento (1994-1995 y 1995-1996)

Como consecuencia, y después de iniciar nuestra fase empírica basada en la observación, en la que se acumuló información sobre eventos o conductas, datos que nos han ido traduciendo la realidad, pasamos a una fase activa donde a partir de nuestro elaborado sistema de categorías, se estableció el instrumento de medida para la investigación observacional.

Dado que entre otras cosas, nuestro objetivo era describir y cuantificar cómo pasaban su tiempo y se repartían el espacio del patio de recreo niños/niñas de la muestra objeto de estudio, los criterios que darán lugar a las unidades de conducta, tienen que ver más que con los acontecimientos, con los estados ${ }^{1}$

El código es una abreviación o símbolo aplicado a un segmento de texto, en nuestro caso, hemos optado en nuestra codificación las siguientes abreviaturas: que se presentan en los cuadros (Cuadros 3 y 4).

Cuadro 3. Unidades de conducta y abreviaturas

\begin{tabular}{|l|l|}
\hline 1.-Fútbol & $\mathrm{F}$ \\
\hline 2.-Comba & $\mathrm{C}$ \\
\hline 3.-Juegos con objeto & $\mathrm{JX}$ \\
\hline 4.-Peonza & $\mathrm{P}$ \\
\hline 5.-Persecuciones & $\mathrm{Pr}$ \\
\hline 6.-Conversaciones & $\mathrm{Co}$ \\
\hline 7.-Agresión & $\mathrm{A}$ \\
\hline 8.-Juegos gráficos & $\mathrm{Jg}$ \\
\hline 9.-Juegos de lucha & $\mathrm{Jl}$ \\
\hline 10.-Juegos de superficie & $\mathrm{Js}$ \\
\hline 11.-Otros & $\mathrm{O}$ \\
\hline
\end{tabular}

\footnotetext{
1 Los acontecimientos corresponden a conductas de relativamente corta duración, como movimientos o vocalizaciones puntuales, y que pueden considerarse como puntos en el tiempo. Los estados poseen una duración apreciable, correspondiendo a actividades prolongadas. La unidad de medida del evento es su frecuencia, y del estado, su duración.
} 
Cantó, R. y Ruiz, L. M. (2005) Comportamiento Motor Espontáneo en el Patio de Recreo Escolar: Análisis de las diferencias por género en la ocupación del espacio durante el recreo escolar. Revista Internacional de Ciencias del Deporte. 1 (1), 28-45. http://www.cafyd.com/REVISTA/art3n1a05.pdf

Cuadro 4. Abreviaturas de cada una de las unidades de conducta por género

\begin{tabular}{|l|l|}
\hline Niños & Niñas \\
\hline F C Jx P Pr Co A Jg Jl Js O & F C Jx P Pr Co A Jg Jl Js O \\
\hline
\end{tabular}

\section{Análisis de los datos}

Una vez recogida toda la información procedente en las hojas de registro durante la fase activa de la investigación, en la que hemos llevado a cabo una observación sistemática y desarrollado cada una de las etapas propias de una metodología observacional, las hojas de registro se distribuyeron de la siguiente manera:

o 40 hojas de registro completadas por el investigador principal (una por sesión), que sumaron un total de 800 minutos de registro de datos. En el cuadro 5, y tablas 1 y 2 se presentan la información global más relevante de los datos recopilados.

Cuadro 5. Resumen de aspectos analizados o unidades manejadas en el estudio

1) Un tiempo total empleado (en minutos) en cada categoría por la totalidad del grupo estudiado, (40 sesiones de 20 minutos de observación = 800 minutos).

2) Un tiempo total por categoría y género.

3) La media de componentes en cada una de las categorías. (Total, niños y niñas).

4) Tablas de significación, en cuanto a las diferencias entre géneros.

5) Densidad de ocupación espacial por categoría en metros cuadrados.

6) Densidad de ocupación espacial por género en la totalidad del patio de recreo escolar, teniendo en cuenta, la media de componentes o participantes por categoría y los metros cuadrados valorados que utilizan por categoría. 
Cantó, R. y Ruiz, L. M. (2005) Comportamiento Motor Espontáneo en el Patio de Recreo Escolar: Análisis de las diferencias por género en la ocupación del espacio durante el recreo escolar. Revista Internacional de Ciencias del Deporte. 1 (1), 28-45. http://www.cafyd.com/REVISTA/art3n1a05.pdf

\section{6.- Resultados}

Tabla 1. Componentes y duración por sesión en cada una de las categorías. Niños

\begin{tabular}{|c|c|c|c|c|c|c|c|c|c|c|}
\hline Sesiones & $\mathrm{F}$ & $\mathrm{C}$ & $\mathrm{Jx}$ & $\operatorname{Pr}$ & Co & $\mathrm{A}$ & $\mathrm{Jg}$ & $\mathrm{Jl}$ & Js & $\mathrm{O}$ \\
\hline 1 & $3-20$ & $2-16$ & $5-15$ & & & & & & & \\
\hline 2 & $7-19$ & $4-16$ & & & & & & & & \\
\hline 3 & $3-18$ & & 3- 16 & & & $2-1$ & & & & \\
\hline 4 & & & $14-19$ & & & & & & & \\
\hline 5 & & & $14-18$ & & & & & & & \\
\hline 6 & & & 14-19 & & & & & & & \\
\hline 7 & 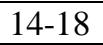 & & & & & & & & & \\
\hline 8 & 5-19 & & $8-19$ & & & & & & & \\
\hline 9 & & $3-18$ & 11-18 & & & & & & & \\
\hline 10 & & & 14-19 & & & & & & & \\
\hline 11 & & & $14-18$ & & & & & & & \\
\hline 12 & $13-19$ & & & & & & & & & \\
\hline 13 & $14-20$ & & & & & & & & & \\
\hline 14 & $10-20$ & & & & & & & $4-20$ & & \\
\hline 15 & $10-20$ & & & $2-3$ & $2-17$ & & & & & \\
\hline 16 & $14-20$ & & & & & $2-1$ & & & & \\
\hline 17 & $10-20$ & & $4-14$ & & & & & & $4-6$ & \\
\hline 18 & $14-20$ & & & & & & & & & \\
\hline 19 & $14-20$ & & & & & & & & & \\
\hline 20 & $14-20$ & & & & & & & & & \\
\hline 21 & $9-20$ & & & $1-20$ & & & & & $5-20$ & \\
\hline 22 & & & $12-20$ & & & & & & & $2-20$ \\
\hline 23 & $3-20$ & & $11-20$ & & & & & & & \\
\hline 24 & $3-20$ & & $11-20$ & & & & & & & \\
\hline 25 & $11-20$ & & $3-20$ & & & & & & & \\
\hline 26 & $10-20$ & & & & & & & & $2-20$ & \\
\hline 27 & $14-20$ & & & & & & & & & \\
\hline 28 & $14-20$ & & & & & & & & & \\
\hline 29 & $8-20$ & & & & & & $2-20$ & & $4-20$ & \\
\hline 30 & $7-20$ & & & 6-15 & & & & & $2-20$ & \\
\hline 31 & $10-20$ & & $4-13$ & & & $2-1$ & & & $4-7$ & \\
\hline 32 & $11-20$ & & & & & $2-1$ & & & $4-20$ & \\
\hline 33 & $9-20$ & & & & & & & & 5-20 & \\
\hline 34 & $2-20$ & & & & $2-8$ & & & & $7-20$ & \\
\hline 35 & $6-20$ & & $8-10$ & & & & & & $8-10$ & \\
\hline 36 & $13-20$ & & $1-20$ & & & & & & & \\
\hline 37 & $12-20$ & & $2-20$ & & & & & & & \\
\hline 38 & $7-20$ & & & & & & & & $6-20$ & \\
\hline 39 & $8-20$ & & & & & & & & $4-20$ & \\
\hline 40 & $12-20$ & & & & & & & & $2-20$ & \\
\hline
\end{tabular}


Cantó, R. y Ruiz, L. M. (2005) Comportamiento Motor Espontáneo en el Patio de Recreo Escolar: Análisis de las diferencias por género en la ocupación del espacio durante el recreo escolar. Revista Internacional de Ciencias del Deporte. 1 (1), 28-45. http://www.cafyd.com/REVISTA/art3n1a05.pdf

Tabla 2. Componentes y duración por sesión en cada una de las categorías. Niñas

\begin{tabular}{|c|c|c|c|c|c|c|c|c|c|c|}
\hline Sesiones & $F$ & C & $\mathrm{Jx}$ & $\mathrm{Pr}$ & Co & A & Jg & $\mathrm{Jl}$ & Js & $\mathrm{O}$ \\
\hline 1 & & $14-16$ & & & $14-16$ & & & & & \\
\hline 2 & & $7-18$ & & & \begin{tabular}{|l|}
$7-18$ \\
\end{tabular} & & & & & $2-17$ \\
\hline 3 & & $14-18$ & & & $14-18$ & & & & & \\
\hline 4 & & $7-18$ & & & $2-18$ & & & & & $5-18$ \\
\hline 5 & & $14-18$ & & & & & & & & \\
\hline 6 & & & & 11-18 & $4-18$ & & & & & \\
\hline 7 & & 13-18 & & & $2-18$ & & & & & $2-6$ \\
\hline 8 & $2-19$ & $3-18$ & & $4-18$ & & & & & & \\
\hline 9 & & $6-18$ & $4-18$ & & & & & & & $4-18$ \\
\hline 10 & & $6-18$ & $7-19$ & & 6-18 & & & & & $2-18$ \\
\hline 11 & & & $2-18$ & & $5-18$ & & & & & $5-18$ \\
\hline 12 & & $7-18$ & 4-18 & & & & & & & \\
\hline 13 & & $10-20$ & $2-9$ & & $4-10$ & & & & & $2-9$ \\
\hline 14 & & $7-20$ & & & $6-20$ & & & & $2-20$ & \\
\hline 15 & & $10-20$ & & $3-3$ & $3-17$ & & & & & \\
\hline 16 & & $14-20$ & & & & & & & & \\
\hline 17 & & $4-7$ & & $10-14$ & $5-6$ & & & & & $5-20$ \\
\hline 18 & & & & & $15-20$ & & & & & \\
\hline 19 & & & & $15-13$ & $8-8$ & & & & & \\
\hline 20 & & 15-6 & & & & & $3-3$ & & & $6-14$ \\
\hline 21 & & & & $6-20$ & $7-20$ & & & & & $7-5$ \\
\hline 22 & & $8-20$ & $2-20$ & & $5-20$ & & & & $2-20$ & \\
\hline 23 & & $13-20$ & & & $2-18$ & & & & & \\
\hline 24 & & $8-11$ & & & $8-9$ & & & & & $2-20$ \\
\hline 25 & & $7-20$ & $2-20$ & 4-13 & $8-20$ & & & & $2-20$ & \\
\hline 26 & $2-7$ & $4-20$ & & & $5-20$ & & & & $2-20$ & \\
\hline 27 & & & & $11-20$ & $2-20$ & & & & $2-20$ & \\
\hline 28 & $2-20$ & & & & $9-10$ & & & & $4-11$ & $11-10$ \\
\hline 29 & & & & & $6-20$ & & & & $2-20$ & $5-20$ \\
\hline 30 & & & & $6-15$ & $6-5$ & & & & & $6-15$ \\
\hline 31 & & & & & $8-13$ & $2-1$ & & & & $2-11$ \\
\hline 32 & & & & & 5-20 & & & & & $8-20$ \\
\hline 33 & & & $5-5$ & & & & & & & $15-15$ \\
\hline 34 & & & & $7-13$ & $7-20$ & & & & & $2-12$ \\
\hline 35 & & & & & $13-20$ & & $2-20$ & & $8-10$ & \\
\hline 36 & & & $6-3$ & $6-2$ & $6-20$ & & & & & \\
\hline 37 & & & $2-20$ & & $7-20$ & & & & & $5-20$ \\
\hline 38 & & & & & $15-20$ & & & & & \\
\hline 39 & & $15-20$ & & & & & & & & \\
\hline 40 & & & & & $13-20$ & & & & & $2-20$ \\
\hline
\end{tabular}


Cantó, R. y Ruiz, L. M. (2005) Comportamiento Motor Espontáneo en el Patio de Recreo Escolar: Análisis de las diferencias por género en la ocupación del espacio durante el recreo escolar. Revista Internacional de Ciencias del Deporte. 1 (1), 28-45. http://www.cafyd.com/REVISTA/art3n1a05.pdf

Densidad media de ocupación espacial por género en el patio de recreo.

Para obtener datos relativos al reparto del espacio entre ambos sexos en sus conductas espontáneas en el patio de recreo escolar, hemos medido las diferentes zonas en las que se desarrollan las conductas categorizadas, y las hemos enfrentado al número total de componentes que ha intervenido en cada una de las categorías de conducta por niños y niñas.

Hay que destacar que a la hora de establecer las mediciones, no todas las zonas o territorialidades, permitian la misma objetividad en el momento de establecer medidas exactas, dado que, por ejemplo, en el campo de Fútbol, como suele ser habitual, viene a estar señalizado por el dibujo del mismo sobre el patio, sin embargo, otras zonas como las de juegos de persecución, han tenido unas medidas, por estimación teniendo en cuenta, la máxima distancia de alejamiento respecto de un punto de origen de la actividad, y a partir de ahí se trazaron las líneas hasta dibujar la zona, al objeto de expresar, la citada territorialidad en metros cuadrados.

En la Tabla 4 se presenta los resultados de la ocupación y reparto del espacio entre niños y niñas, de tal manera que se puede confirmar que la densidad / metro cuadrado de las niñas fue de $\mathbf{5 8 , 1 5}$ y en los niños de $\mathbf{2 2 , 8 5}$. Si tan solo tenemos en cuenta que el grupo lo componen 15 niñas y 14 niños, la desigual en ocupación y uso del espacio es clara, los varones utilizan más del doble del espacio disponible que las niñas. (Tablas 3, Fig. 1)

Tabla 3. Estadísticos descriptivos de la muestra total por categoría

Estadísticos descriptivos de la muestra total

\begin{tabular}{|c|c|c|c|c|c|c|}
\hline & \multirow[b]{2}{*}{$\mathrm{N}$} & \multirow[b]{2}{*}{ Media } & \multirow[b]{2}{*}{$\begin{array}{l}\text { Desviación } \\
\text { típica }\end{array}$} & \multicolumn{3}{|c|}{ Percentiles } \\
\hline & & & & 25 & $\begin{array}{c}50 \\
\text { (Mediana) }\end{array}$ & 75 \\
\hline $\begin{array}{l}\text { Comba } \\
\left(\mathrm{comps} / \mathrm{m}^{2}\right)\end{array}$ & 80 & .1005 & .2060 & .0000 & .0000 & 3.698E-02 \\
\hline $\begin{array}{l}\text { Conversaciones } \\
\left(\mathrm{comps} / \mathrm{m}^{2}\right)\end{array}$ & 80 & .4545 & .6517 & .0000 & .0000 & .8141 \\
\hline $\begin{array}{l}\text { Fútbol } \\
(\text { comps/m²) }\end{array}$ & 80 & .2105 & .2586 & .0000 & .0000 & .4418 \\
\hline $\begin{array}{l}\text { J.Gráficos } \\
(\text { comps/m²) }\end{array}$ & 80 & 1.761E-03 & 1.575E-02 & .0000 & .0000 & .0000 \\
\hline $\begin{array}{l}\text { J.Objeto } \\
\text { (comps/m²) }\end{array}$ & 80 & .1072 & .2034 & .0000 & .0000 & .1356 \\
\hline $\begin{array}{l}\text { Otros } \\
(\text { comps/m²) }\end{array}$ & 80 & 6.136E-02 & .1296 & .0000 & .0000 & $9.818 \mathrm{E}-02$ \\
\hline $\begin{array}{l}\text { J.Persec } \\
(\text { comps/m²) }\end{array}$ & 80 & 5.671E-02 & .1517 & .0000 & .0000 & .0000 \\
\hline $\begin{array}{l}\text { J.Superficie } \\
(\text { comps/m²) }\end{array}$ & 80 & 6.994E-02 & .1410 & .0000 & .0000 & .1190 \\
\hline Niños-as/m² & 80 & 1.0625 & .5332 & .6897 & .8615 & 1.2908 \\
\hline
\end{tabular}


Cantó, R. y Ruiz, L. M. (2005) Comportamiento Motor Espontáneo en el Patio de Recreo Escolar: Análisis de las diferencias por género en la ocupación del espacio durante el recreo escolar. Revista Internacional de Ciencias del Deporte. 1 (1), 28-45. http://www.cafyd.com/REVISTA/art3n1a05.pdf

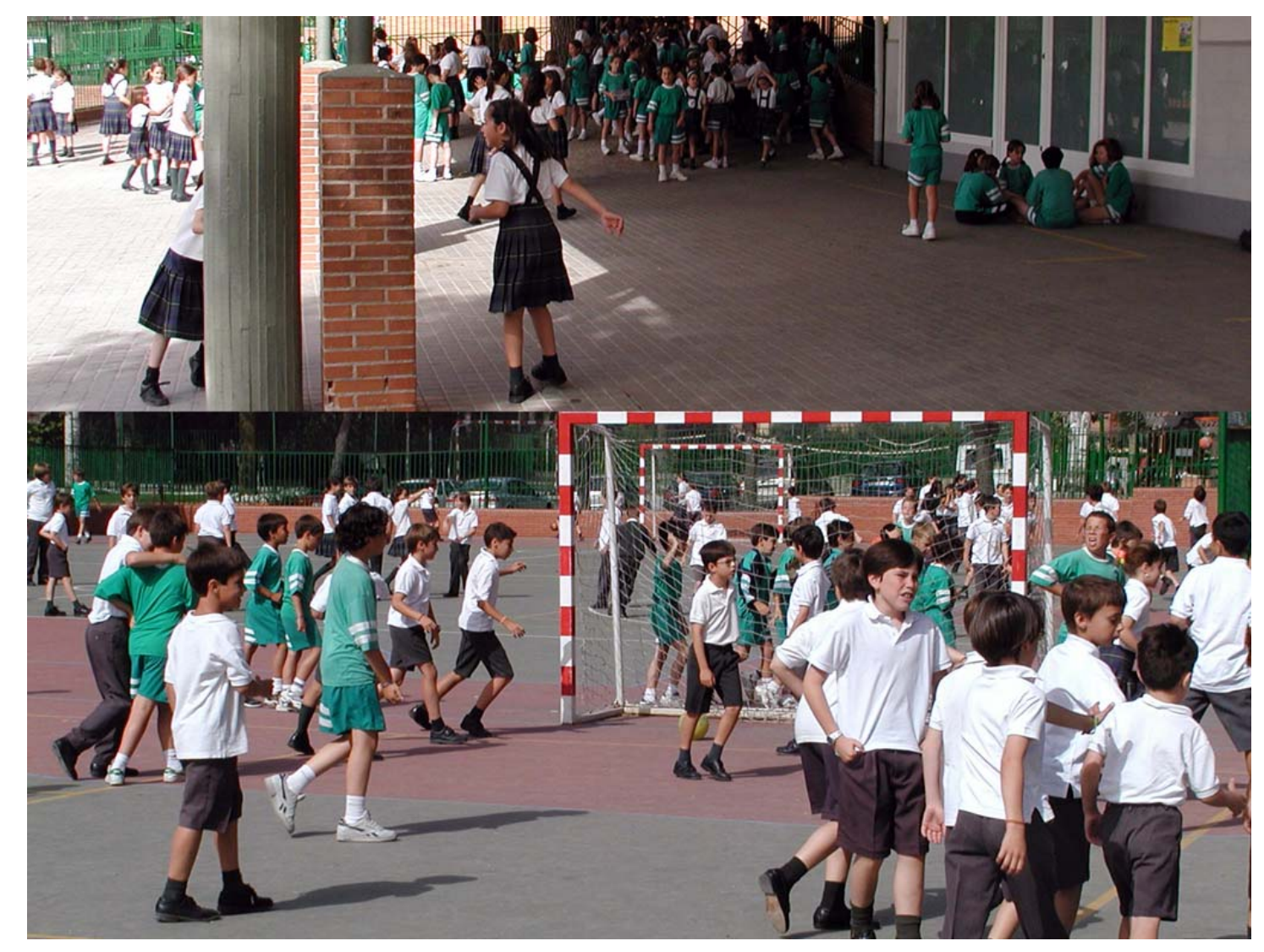

Fig. 1 
Cantó, R. y Ruiz, L. M. (2005) Comportamiento Motor Espontáneo en el Patio de Recreo Escolar: Análisis de las diferencias por género en la ocupación del espacio durante el recreo escolar. Revista Internacional de Ciencias del Deporte. 1 (1), 28-45. http://www.cafyd.com/REVISTA/art3n1a05.pdf

Tabla 4. Estadísticos descriptivos de categorías según género

Estadísticos descriptivos según el género

\begin{tabular}{|c|c|c|c|c|c|c|c|}
\hline \multirow[b]{2}{*}{ Sexo } & & \multirow{2}{*}{$\frac{\mathrm{N}}{\mathrm{N}}$} & \multirow[b]{2}{*}{ Media } & \multirow[b]{2}{*}{ Desv. típ. } & \multicolumn{3}{|c|}{ Percentiles } \\
\hline & & & & & 25 & 50 & 75 \\
\hline \multirow[t]{9}{*}{ Niñas } & $\begin{array}{l}\text { Comba } \\
\left(\text { comps } / \mathrm{m}^{2}\right)\end{array}$ & 40 & 1935 & ,2596 & ,0000 & ,0000 & ,3452 \\
\hline & $\begin{array}{l}\text { Conversaciones } \\
\left(\text { comps } / \mathrm{m}^{2}\right)\end{array}$ & 40 & ,8955 & ,6765 & ,2714 & ,8141 & 1,4925 \\
\hline & $\begin{array}{l}\text { Fútbol } \\
(\text { comps/m²) }\end{array}$ & 40 & ,0356 & ,1019 & ,0000 & ,0000 & ,0000 \\
\hline & $\begin{array}{l}\text { J.Gráficos } \\
\left(\text { comps } / \mathrm{m}^{2}\right)\end{array}$ & 40 & ,0035 & ,0223 & ,0000 & ,0000 & ,0000 \\
\hline & $\begin{array}{l}\text { J.Objeto } \\
\text { (comps/m²) }\end{array}$ & 40 & ,0345 & ,0859 & ,0000 & ,0000 & ,0000 \\
\hline & $\begin{array}{l}\text { Otros } \\
\left(\text { comps } / \mathrm{m}^{2}\right)\end{array}$ & 40 & 1178 & 1644 & ,0000 & ,0982 & 1964 \\
\hline & $\begin{array}{l}\text { J.Persec } \\
\left(\text { comps } / \mathrm{m}^{2}\right)\end{array}$ & 40 & 1097 & ,2015 & ,0000 & ,0000 & 1849 \\
\hline & $\begin{array}{l}\text { J.Superficie } \\
\left(\text { comps } / \mathrm{m}^{2}\right)\end{array}$ & 40 & ,0268 & ,0873 & ,0000 & ,0000 & ,0000 \\
\hline & Total comps $/ \mathrm{m}^{2}$ & 40 & 1,4170 & ,5476 & ,9761 & 1,2877 & 1,8902 \\
\hline \multirow[t]{9}{*}{ Niños } & $\begin{array}{l}\text { Comba } \\
\left(\text { comps } / m^{2}\right)\end{array}$ & 40 & ,0074 & ,0286 & ,0000 & ,0000 & ,0000 \\
\hline & $\begin{array}{l}\text { Conversaciones } \\
\left(\mathrm{comps} / \mathrm{m}^{2}\right)\end{array}$ & 40 & ,0136 & ,0599 & ,0000 & ,0000 & ,0000 \\
\hline & $\begin{array}{l}\text { Fútbol } \\
\text { (comps/m²) }\end{array}$ & 40 & ,3854 & ,2497 & , 1473 & ,4418 & ,6259 \\
\hline & $\begin{array}{l}\text { J.Gráficos } \\
\left(\text { comps } / \mathrm{m}^{2}\right)\end{array}$ & 40 & ,0000 & ,0000 & & & \\
\hline & $\begin{array}{l}\text { J.Objeto } \\
\left(\text { comps } / \mathrm{m}^{2}\right)\end{array}$ & 40 & 1800 & ,2560 & ,0000 & ,0000 & ,3575 \\
\hline & $\begin{array}{l}\text { Otros } \\
\left(\text { comps } / \mathrm{m}^{2}\right)\end{array}$ & 40 & ,0049 & ,0217 & ,0000 & ,0000 & ,0000 \\
\hline & $\begin{array}{l}\text { J.Persec } \\
\left(\text { comps } / \mathrm{m}^{2}\right)\end{array}$ & 40 & ,0037 & ,0173 & ,0000 & ,0000 & ,0000 \\
\hline & $\begin{array}{l}\text { J.Superficie } \\
\left.\text { (comps } / m^{2}\right)\end{array}$ & 40 & , 1131 & 1699 & ,0000 & ,0000 & ,2381 \\
\hline & Total comps $/ \mathrm{m}^{2}$ & 40 & ,7080 & ,1352 & ,6873 & ,6900 & ,7368 \\
\hline
\end{tabular}

Para establecer las diferencias entre las diferentes actividades en chicos y chicas se llevó a cabo la prueba de Kolmogorov y Smirnov para una sola muestra, la prueba de rangos y el U de Man- Whitney cuyos resultados se presentan en las tablas5, 6 y 7. 
Cantó, R. y Ruiz, L. M. (2005) Comportamiento Motor Espontáneo en el Patio de Recreo Escolar: Análisis de las diferencias por género en la ocupación del espacio durante el recreo escolar. Revista Internacional de Ciencias del Deporte. 1 (1), 28-45. http://www.cafyd.com/REVISTA/art3n1a05.pdf

Tabla 5. Resultados de la prueba de Kolmogorov y Smirnov

Prueba de Kolmogorov-Smirnov para una muestra

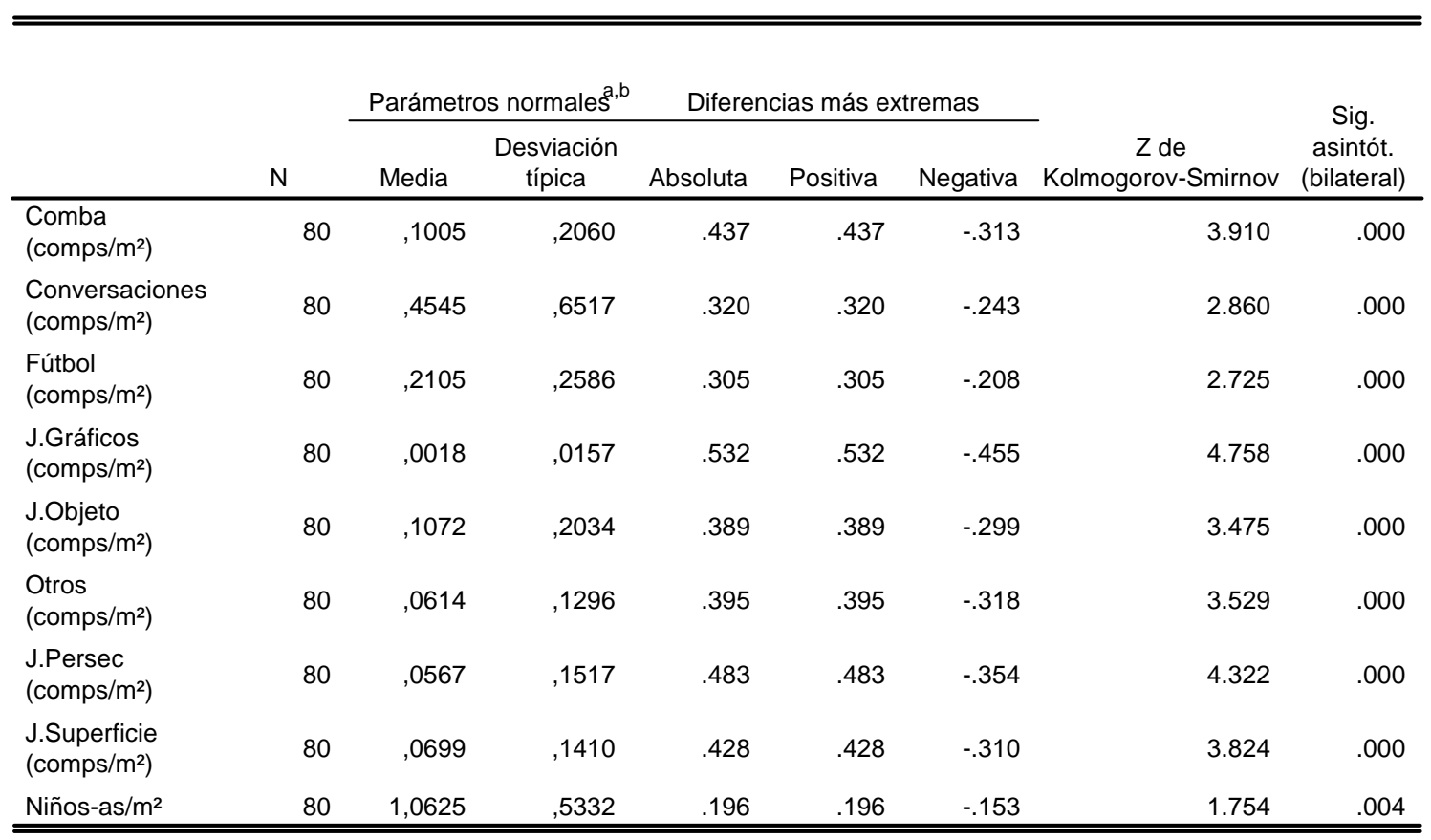

a. La distribución de contraste es la Normal.

b. Se han calculado a partir de los datos. 
Cantó, R. y Ruiz, L. M. (2005) Comportamiento Motor Espontáneo en el Patio de Recreo Escolar: Análisis de las diferencias por género en la ocupación del espacio durante el recreo escolar. Revista Internacional de Ciencias del Deporte. 1 (1), 28-45. http://www.cafyd.com/REVISTA/art3n1a05.pdf

Tabla 6. Prueba de Rangos niños y niñas por categoría

\section{Rangos}

\begin{tabular}{|c|c|c|c|c|}
\hline & Sexo & $\mathrm{N}$ & $\begin{array}{c}\text { Rango } \\
\text { promedio }\end{array}$ & $\begin{array}{c}\text { Suma de } \\
\text { rangos }\end{array}$ \\
\hline \multirow{3}{*}{$\begin{array}{l}\text { Comba } \\
\left(\mathrm{comps} / \mathrm{m}^{2}\right)\end{array}$} & Niñas & 40 & 48.13 & 1925.00 \\
\hline & Niños & 40 & 32.88 & 1315.00 \\
\hline & Total & 80 & & \\
\hline \multirow{3}{*}{$\begin{array}{l}\text { Conversaciones } \\
\left(\mathrm{comps} / \mathrm{m}^{2}\right)\end{array}$} & Niñas & 40 & 56.72 & 2269.00 \\
\hline & Niños & 40 & 24.27 & 971.00 \\
\hline & Total & 80 & & \\
\hline \multirow{3}{*}{$\begin{array}{l}\text { Fútbol } \\
(\text { comps/m²) }\end{array}$} & Niñas & 40 & 25.33 & 1013.00 \\
\hline & Niños & 40 & 55.67 & 2227.00 \\
\hline & Total & 80 & & \\
\hline \multirow{3}{*}{$\begin{array}{l}\text { J.Gráficos } \\
\text { (comps/m²) }\end{array}$} & Niñas & 40 & 41.00 & 1640.00 \\
\hline & Niños & 40 & 40.00 & 1600.00 \\
\hline & Total & 80 & & \\
\hline \multirow{3}{*}{$\begin{array}{l}\text { J.Objeto } \\
\left(\text { comps } / \mathrm{m}^{2}\right)\end{array}$} & Niñas & 40 & 34.26 & 1370.50 \\
\hline & Niños & 40 & 46.74 & 1869.50 \\
\hline & Total & 80 & & \\
\hline \multirow{3}{*}{$\begin{array}{l}\text { Otros } \\
\text { (comps/m²) }\end{array}$} & Niñas & 40 & 50.30 & 2012.00 \\
\hline & Niños & 40 & 30.70 & 1228.00 \\
\hline & Total & 80 & & \\
\hline \multirow{3}{*}{$\begin{array}{l}\text { J.Persec } \\
(\text { comps/m²) }\end{array}$} & Niñas & 40 & 45.28 & 1811.00 \\
\hline & Niños & 40 & 35.72 & 1429.00 \\
\hline & Total & 80 & & \\
\hline \multirow{3}{*}{$\begin{array}{l}\text { J.Superficie } \\
\left(\text { comps } / \mathrm{m}^{2}\right)\end{array}$} & Niñas & 40 & 34.65 & 1386.00 \\
\hline & Niños & 40 & 46.35 & 1854.00 \\
\hline & Total & 80 & & \\
\hline \multirow[t]{3}{*}{ Niños-as/m² } & Niñas & 40 & 58.15 & 2326.00 \\
\hline & Niños & 40 & 22.85 & 914.00 \\
\hline & Total & 80 & & \\
\hline
\end{tabular}


Cantó, R. y Ruiz, L. M. (2005) Comportamiento Motor Espontáneo en el Patio de Recreo Escolar: Análisis de las diferencias por género en la ocupación del espacio durante el recreo escolar. Revista Internacional de Ciencias del Deporte. 1 (1), 28-45. http://www.cafyd.com/REVISTA/art3n1a05.pdf

Tabla 7. Resultados de la prueba U de Mann-Whitney

Estadísticos de contraste

\begin{tabular}{|c|c|c|c|}
\hline & $\begin{array}{c}\text { U de } \\
\text { Mann-Whitney }\end{array}$ & Z & $\begin{array}{c}\text { Sig. } \\
\text { asintót. } \\
\text { (bilateral) }\end{array}$ \\
\hline $\begin{array}{l}\text { Comba } \\
\left(\mathrm{comps} / \mathrm{m}^{2}\right)\end{array}$ & 495.000 & -3.860 & .000 \\
\hline $\begin{array}{l}\text { Conversaciones } \\
\left(\mathrm{comps} / \mathrm{m}^{2}\right)\end{array}$ & 151.000 & -6.892 & .000 \\
\hline $\begin{array}{l}\text { Fútbol } \\
(\text { comps/m²) }\end{array}$ & 193.000 & -6.285 & .000 \\
\hline $\begin{array}{l}\text { J.Gráficos } \\
(\text { comps/m²) }\end{array}$ & 780.000 & -1.000 & .317 \\
\hline $\begin{array}{l}\text { J.Objeto } \\
(\text { comps/m²) }\end{array}$ & 550.500 & -2.923 & .003 \\
\hline $\begin{array}{l}\text { Otros } \\
(\text { comps/m²) }\end{array}$ & 408.000 & -4.731 & .000 \\
\hline $\begin{array}{l}\text { J.Persec } \\
\left(\mathrm{comps} / \mathrm{m}^{2}\right)\end{array}$ & 609.000 & -2.861 & .004 \\
\hline $\begin{array}{l}\text { J.Superficie } \\
(\text { comps/m²) }\end{array}$ & 566.000 & -2.911 & .004 \\
\hline Total comps/m2 & 94.000 & -6.799 & .000 \\
\hline
\end{tabular}

a. Variable de agrupación: Sexo

Aunque el resultado que nos interesa, es el reparto global del espacio disponible en cuanto a su ocupación y uso en los comportamientos espontáneos entre niños y niñas, y que ya hemos podido constatar arroja una diferencia significativa, cabe señalar que la única categoría que no establece diferencia, es la categoría Juego Gráfico. No debe olvidarse, que los diferentes espacios son enfrentados a la densidad de ocupación por ambos géneros.

Tras haber medido las diferentes zonas en las que se desarrollan las conductas categorizadas, y enfrentado al número total de componentes que ha intervenido en cada una de las categorías de conducta por niños y niñas. A pesar de la relativa objetividad que planteaban algunas zonas a la hora de ser medidas en torno a un núcleo categorial en concreto, sobre todo a la hora de medir territorialidades con líneas imaginarias, mientras otros, nos referimos a los campos de fútbol, estaban marcados y señalizados.

A la hora de trazar un zona para medir, por ejemplo en el lugar donde se desarrollaba la actividad de la Comba, sabíamos que la objetividad no era la misma, extremo que se complicaba, si mediamos la zona en la que se desarrollaban juegos de persecución, a pesar de eso, y conscientes del margen de error que ello llevaba implícito, continuamos con nuestra labor de medir, pues bien, a la hora de ver los resultados, nuestro alivio a sido grande al comprobar un contraste tan grande, que en ninguno de los casos podia achacarsele a la citada dispar objetividad con la que decidimos medir las distintas territorialidades adujudicadas a cada una de las categorías. 
El interrogante que se nos plantea inmediatamente es si realmente es tan desigual el reparto del espacio disponible, si este reparto está negociado de antemano, y si los varones sería capaces de aceptar un reparto diferente al observado en este estudio En una interesante ponencia realizada por Asins en 1992, sobre la ocupación diferencial del espacio en Educación física, nos dice que las implicaciones del comportamiento del ser humano con relación al espacio no resultan sencillas de comprender, desde muy diversos ámbitos del conocimiento se han investigado diferentes aspectos de esta interrelación; Antropología, Sociología, Geografía, Psicología, Etología, Arquitectura y Urbanismo le han dedicado una buena producción de sus trabajos.

Desde sus aportaciones nos muestran la riqueza y complejidad que se esconde en algo tan cotidiano, y por ello, en ocasiones tan oculto e inconsciente como es nuestra ocupación y utilización del espacio.

Hemos encontrado de interés la afirmación de que está demostrado que en el medio urbano el sexo, junto con el nivel socioconómico, y la edad, son variables que condicionan la movilidad espacial, y debemos recordar que en una sociedad donde los ámbitos de residencia, producción y servicios están disociados espacialemente, el acceso al mercado de trabajo y a los servicios es tanto pero cuanto más limitada sea esa movilidad. Y el espacio es un elemento más de socialización y los estereotipos sexuales limitan y condicionan considerablemente la movilidad espacial de las niñas.

\section{Discusión}

Los resultados, que nos han permitido concluir con la afirmación de que los niños y niñas de la muestra estudiada desarrollan un comportamiento diferenciado, nos han permitido también, sumarnos a la larga lista de los que vienen manteniendo estas diferencias de género. A la hora de presentar de forma ordenada los registros, y observando cómo se distribuyen, puede tenerse una inicial apreciación visual de la existencia de una diferencia significativa en el comportamiento espontáneo de ambos géneros en el patio de recreo escolar

Este estudio ha mostrado que existe una diferencia en el comportamiento espontáneo entre géneros cuando se encuentran en el recreo descrito en esta investigación, y aunque, se dedicaron muchas horas en la fase de observación reflexión de nuestra investigación a los registros narrativos, aún así, la espontaneidad de la que presumíamos inicialmente, se ha impregnado de cierta relatividad en relación con alguno de los núcleos categoriales, nos estamos refiriendo a algo que fue cobrando importancia en la medida que avanzábamos en nuestro estudio, algo que quedó bastante patente cuando se comprobó el dato referido a la densidad de población por metro cuadrado y genero, a la hora de ocupar los escolares el espacio disponible del patio de recreo, tal desigual reparto del espacio venia a confirmar, lo que se ha convenido en llamar, espacios inductores, y por tanto reductores de espontaneidad en las conductas, concretamente, nos estamos preguntando, si el dato de referencia, lo que ha permitido afirmar: que se ha dado un mal reparto del espacio, el constatar que mientras los niños han mantenido una densidad de media de 22,85 individuos, las niñas la han tenido de un 58, 15 individuos del total del espacio ocupado, y se debe dejar de tener presente que se trató de una muestra formada por 14 niños y 15 niñas. 
Y también nos preguntamos, si el predominio de las conductas relacionadas con el núcleo categorial Fútbol, sería el mismo, de no tener una presencia tan tangible, un campo de fútbol señalizado, delimitado y con porterías, ocupando un gran porcentaje del total del patio de recreo escolar.

\section{Conclusiones}

Como consideraciones finales de este estudio podemos decir que los niños han realizado una ocupación y uso desigual del espacio disponible en el recreo escolar, respecto de las niñas. Aparte de la apreciación visual que evidencia como grandes concentraciones de niñas, se juntan en torno a reducidos espacios, puede apreciarse como reducidos grupos de niños se distribuyen en grandes espacios (ejemplo de un campo de fútbol, que a veces ocupan la mayor parte del espacio disponible).

Esta apreciación deja de serlo en el momento que utilizamos los datos obtenidos, para ser una afirmación, ya que los niños ocupan y utilizan más espacio que las niñas. Tras haber adjudicado, a posteriori, un espacio medido en metros cuadrados a cada una de las categorías de conducta desarrolladas espontáneamente, y a la vista de el número de componentes por categoría y género, hemos concluido en la densidad / metro cuadrado de ocupaciòn de las niñas fue de 58,15 y en los niños de 22,85.

Dato que nos permite concluir o expresar el hecho, en términos de mal reparto de género del espacio disponible, sin entrar en problemas de equidad o desigualdad de oportunidades, extrapolables a planteamientos de futuro (otros niveles educativos, universidad, ámbito laboral, etc.). 


\section{Referencias bibliográficas}

Álvarez. C. Reflexiones en torno a la participación de las mujeres en la ciencia y la tecnología. Artículo de referencia: Ciencia, Tecnología y Género. Marta I. González García y Eulalia Pérez Sedeño. (2002). Revista Ibero Americana de Ciencia y Tecnología 2. Abril de 2002

Anguera, M. T. (1988). Observación en la escuela. Barcelona: Grao.

Asins Muñoz, C. (1994). La ocupación diferencial del espacio en educación física. L'Ensenyament de L'Educació Física. Ponencias IV Jornadas Internacionales de Coeducación. Valencia 437-448

Blurton Jones, N. G. (1967). An Ethological Study of some aspects of Behaviour od children in Nursey School. New York: Anchor Books.

Brullet, C. Subirats, M (1988). La transmisión de lo géneros en la escuela mixta. Serie Rosa y Azul. Ministerio de Cultura. Instituto de la Mujer.

Canto, R. (2004) Comportamiento motor espontáneo en el patio de recreo escolar. Tesis Doctoral Inédita. Madrid: Universidad Politécnica de Madrid

Delgado, M. A. Y del Villar, F. (1995) El análisis del contenido en la investigación de la enseñanza de la Educación Física. Motricidad, 1, 25-43

García, M. y. Asins, C. (1994b). La coeducación en educación física. Barcelona: Instituto de Ciencias de la Educación.

Grugeon, E. (1995). Implicaciones de género en la cultura del patio de recreo. En Woods, P. y otros 1995(compiladores). Barcelona: Paidós.

Hall, E. T. (1973). La dimensión oculta. Un enfoque antropológico del uso del espacio. (Vol. Nuevo urbanismo 6). Madrid: Instituto de estudios de la administración local.

Pomerleau, A. y M., G. (1992). El niño y su ambiente. (1a ed.). México: Trillas. 